\title{
Traditional Chinese Medicine Decreases the Stroke Risk of Systemic Corticosteroid Treatment in Dermatitis: A Nationwide Population-Based Study
}

\author{
Kao-Sung Tsai,, ${ }^{1,23}$ Chia-Sung Yen, ${ }^{3}$ Po-Yuan Wu, ${ }^{1,2}$ Jen-Huai Chiang, ${ }^{1,2}$ Jui-Lung Shen, \\ Chung-Hsien Yang, ${ }^{1}$ Huey-Yi Chen, ${ }^{1,2}$ Yung-Hsiang Chen, ${ }^{1,2,6}$ and Wen-Chi Chen ${ }^{1,2}$ \\ ${ }^{1}$ Institute of Chinese Medicine, School of Chinese Medicine, Graduate Institute of Integrated Medicine, \\ School of Post-Baccalaureate Chinese Medicine, Research Center for Chinese Medicine \& Acupuncture, \\ Institute of Clinical Medical Science, College of Medicine, China Medical University, Taichung 40402, Taiwan \\ ${ }^{2}$ Departments of Dermatology, Medical Research, Obstetrics and Gynecology, and Urology, Management Office for Health Data, China \\ Medical University Hospital, Taichung 40447, Taiwan \\ ${ }^{3}$ Department of Applied Cosmetology, Master Program of Cosmetic Science, and Department of Cultural and Creative Industries, \\ Hungkuang University, Taichung 43302, Taiwan \\ ${ }^{4}$ Center for General Education, Feng Chia University, Taichung 40724, Taiwan \\ ${ }^{5}$ Department of Dermatology, Taichung Veterans General Hospital, Taichung 40705, Taiwan \\ ${ }^{6}$ Department of Psychology, College of Medical and Health Science, Asia University, Taichung 41354, Taiwan
}

Correspondence should be addressed to Yung-Hsiang Chen; yhchen@mail.cmu.edu.tw and Wen-Chi Chen; wgchen@mail.cmu.edu.tw

Received 4 March 2015; Accepted 15 June 2015

Academic Editor: Cheryl Hawk

Copyright (c) 2015 Kao-Sung Tsai et al. This is an open access article distributed under the Creative Commons Attribution License, which permits unrestricted use, distribution, and reproduction in any medium, provided the original work is properly cited.

\begin{abstract}
Epidemiological studies have shown a strong association between dermatitis and stroke. Systemic corticosteroid, the mainstay treatment for dermatitis, could enhance the atherosclerotic process. Traditional Chinese Medicine (TCM) has been used for dermatitis to decrease the side effects of corticosteroid. However, the different stroke risk in dermatitis patients treated with systemic corticosteroid or TCM remains unclear. This study identified 235,220 dermatitis patients and same comorbidity matched subjects between 2000 and 2009 from database of NHRI in Taiwan. The two cohorts were followed until December 31, 2011. The primary outcome of interest was new diagnosis of stroke. The crude hazard ratio (HR) for future stroke among dermatitis patients treated with systemic corticosteroid was 1.40 (95\% CI, 1.34-1.45; $P<0.0001)$ and TCM was 1.09 (95\% CI, 1.05-1.13; $P<0.0001)$. The log-rank test showed a higher cumulative incidence of ischemic stroke in the patient treated with only systemic corticosteroid group than that treated with systemic corticosteroid and TCM, only TCM, and neither systemic corticosteroid nor TCM in the matched cohort during the follow-up period $(P<0.0001)$. We demonstrated that patients treated with systemic corticosteroid had an increased risk of stroke and that the risk probably decreased by TCM treatment.
\end{abstract}

\section{Introduction}

Many complementary and alternative medicine (CAM) practices have emphasized health promotion; however, this has not been the focus of the bulk of CAM research. CAM practitioners could be seen as a public health resource to increase the population's access to certain clinical preventive services $[1,2]$. Eczematous dermatoses account for a large proportion of all skin disease. Some studies have suggested that dermatitis is an allergic disease in which systemic inflammation involves more than just the skin [3-5]. More evidences have shown that systemic inflammation can accelerate the progression of atherosclerosis and thrombosis with resulting ischemic stroke [6]. Epidemiological studies have shown a strong association between systemic inflammatory disease, particularly dermatoses, and cardiovascular diseases 
[7]. Furthermore, Su et al. demonstrated that atopic dermatitis, a chronically relapsing and constitutive skin disease, may be an independent risk factor for ischemic stroke [8].

Contemporary medicines often used combinations of topical steroid agents, systemic antihistamine, corticosteroids, and immune-modulating agents to control this frustrating disease. The treatment of dermatitis, especially systemic corticosteroid therapy, can influence the atherosclerotic process. It is believed that this treatment is atherogenic for the long-term used, partially due to effects on plasma lipoproteins, elevation of total cholesterol and triglycerides and for promoting an abnormal distribution of high-density lipoprotein subclasses [9]. The systemic corticosteroid can also indirectly accelerate the process by augmenting other traditional risk factors, including hypertension and obesity [10]. On the other hand, inflammation is associated with atherosclerosis, and therefore corticosteroid therapy could have a protective effect. Previous studies published in the literature about this issue were contradictory. The role of treatment with systemic corticosteroid or alterative treatment in the evolution of stroke in dermatitis need to be further investigated.

The decision to use CAM is multifactorial, including dissatisfaction with conventional treatment and frustration with the chronic nature course of eczema. For avoiding the potential adverse effects of systemic conventional dermatitis treatments and also to attain better clinical outcomes, many patients and practitioners have tried to seek alternative treatment [11]. Regarding the benefits, there is a raising trend of CAM treatment and the use of CAM is actually associated with eczema prevalence [12]. Traditional Chinese Medicine (TCM) is one of the popular alternative treatments for dermatitis in Asia and the world $[13,14]$. The aim of this study was to determine the different risk of stroke in dermatitis patients treated with systemic corticosteroid or TCM by using a nationwide database and proved a part of a structured initiative to established evidenced-based clinical recommendation for management of comorbidities in dermatitis.

\section{Materials and Methods}

2.1. Data Sources. Taiwan's National Health Insurance (NHI) program, implemented by the government in March 1995, provides comprehensive health care to almost all Taiwanese citizens, with a coverage rate of more than $99 \%$ of Taiwan's entire population, and contracted with $97 \%$ of hospitals and $92 \%$ of clinics. The National Health Research Institute (NHRI) of Taiwan manages and publicly releases for research purposes multiple NHI databases that include information about basic patient characteristics, date of visit, diagnoses codes for the International Classification of Diseases, Ninth Revision, Clinical Modification (ICD-9-CM) codes, detailed claims data for examinations, disease management, and drug prescriptions for all admitted patients and outpatients $[15,16]$. The NHRI created research data sets including a random sample of 1,000,000 subjects from the registry of all NHI enrollees in 2000, with the encryption of personal information that could identify any individual patient. We obtained these data sets of NHRI from 2000 to 2011 for use as our research database. This study was approved by the Institutional Review Board of CMU-REC-101-012 from Institutional Review Board approval of Public Health, Social and Behavioral Science Committee Research Ethics Committee, China Medical University and Hospital.

2.2. Study Design and Population. This population-based cohort study utilizing a nationwide database was conducted of two groups. The population with dermatitis (aged $\geq 20$ years) were identified by codes 690.X, 691.X, and 692.X in the ICD-9-CM and newly dermatitis diagnosis (at least two medical visits) between 1 January 2000 and 31 December 2009 and followed up until December 31, 2011. Subjects who have a past history of stroke before the enrollment date were excluded from the study group. Systemic corticosteroid or TCM coding was obtained for medication variant control in advanced step of analysis. We included the most common prescribed systemic corticosteroids: dexamethasone, methylprednisolone, and prednisolone. Treatment was divided into non-TCM and nonsystemic steroid user, only TCM user, only systemic steroid user, and TCM and systemic steroid user. The primary outcome of interest was new diagnosis of stroke (ICD-9-CM codes: 430-438). For stroke type analysis, we separated hemorrhagic stroke (ICD-9-CM codes 430, 431, and 432) and compared the ischemic stroke (ICD-9-CM codes 433-438) in further adjusted hazard ratio analysis. The date for dermatitis diagnosis was defined as index date. All the subjects were followed from the index date to occurrence of endpoint or until December 31, 2011, whichever was first, and the observations on the last dates were considered as censored observations.

2.3. Comparison Group. Subjects without dermatitis were randomly selected from the same data set. Each patient with newly diagnosed dermatitis in the NHRI database was pairmatched with one subject of the same age, sex, and index year. TCM or systemic corticosteroid medications and comorbidities (allergic rhinitis, asthma, urticarial, diabetes mellitus, hypertension, hyperlipidemia, and atrial fibrillation) were not matched. We selected comparison subjects using incidence density sampling by computer programming [17]. In the comparison group, subjects who have past history of stroke before enrollment were also excluded as the study group.

To determine stroke and survival analyses adjusting for age, sex, comorbidities, and medications were carried out with Cox's proportional hazards model. All enrollees were followed from the date of enrollment until the first diagnosis of stroke or censored date of death, or date of withdrawal from the insurance, or until 31 December 2011.

2.4. Potential Confounders. In the analysis of the effect of different treatment, systemic corticosteroid or TCM, in patients with dermatitis on the outcome of stroke, we controlled the age and sex and identified the following comorbidities as potential confounders: diabetes mellitus (ICD-9-CM code: 250), hypertension (ICD-9-CM codes: 401-405), hyperlipidemia (ICD-9-CM codes: 272.0, 272.1, 272.2, 272.3, and 272.4), and atrial fibrillation (ICD-9-CM code: 427.31). 
2.5. Statistical Analysis. Person-years of two populations were calculated from baseline to the occurrence of stroke or closing date (December 31, 2011). All statistical analyses were performed using SAS version 9.4 software (SAS Institute, Inc., Cary, NC).

All data are expressed as mean standard deviation or $n(\%)$ unless otherwise stated. Comparisons between groups were performed using Student's $t$-test for continuous variables and Pearson's chi-square test, as appropriate, for categorical variables. The Cox's proportional hazards model was used to estimate the hazards ratio for the progression of outcome. The probability of survival difference between each group with dermatitis user and nondermatitis users was tested with the log-rank test. The Kaplan-Meier method was used to plot the cumulative incidence. Cox proportional hazard model was used to calculate the hazard ratios and $95 \%$ confidence interval of stroke for patients with dermatitis compared with nondermatitis user. All analyses were carried out with SAS statistical software. All statistical tests were performed at the two-tailed significance level of 0.05 . A $P$ value $<0.05$ was considered statistically significant.

\section{Results}

Clinical characteristics of this study population identified patients newly diagnosed with dermatitis between 1 January 2000 and 31 December 2009. After excluding patients aged under 20 years or with antecedent stroke, 235,220 patients with dermatitis were included in the analyses. Other 235,220 patients without dermatitis were selected by $1: 1$ matching by age, sex, and index year. The study subjects were predominantly female $(58.13 \%)$, and the median age was $41.9 \pm$ 15.5 years for dermatitis cohort group and $41.5 \pm 15.9$ years for nondermatitis cohort group. Table 1 shows that basic characteristics and selected comorbidities were similar between groups.

Predictors of difference stroke risk between systemic corticosteroid and TCM treatment in patients with dermatitis were conducted in this study. During the follow-up period, $206,402(87.75 \%)$ patients with dermatitis were treated with systemic corticosteroid and 160,541 (68.25\%) were comparison subjects. $207,890(88.38 \%)$ patients with dermatitis treated with TCM and 183,949 (78.20\%) were comparison subjects. Also, subject with and without dermatitis had 78.47 and 57.22 percentage who had used both TCM and systemic steroid. We also found that $13,079(5.65 \%)$ patients with dermatitis and 10,006 (4.25\%) comparison subjects experienced stroke attack. Analyzing different stroke type, $12,450(5.29 \%)$ patients with dermatitis and 9,277 (3.94\%) comparison subjects had Ischemic stroke attack. However, there was no statistically difference in patients with dermatitis and comparison subjects that experienced hemorrhagic stroke attack. The log-rank test showed a higher cumulative incidence of stroke in the dermatitis group than in the matched cohort during the follow-up period $(P<0.0001$, Figure 1), suggesting that patients with dermatitis had an increased risk of stroke in the long term.

After adjusting for age, gender, comorbidities, and medications, we compared with comparison subjects and stratified

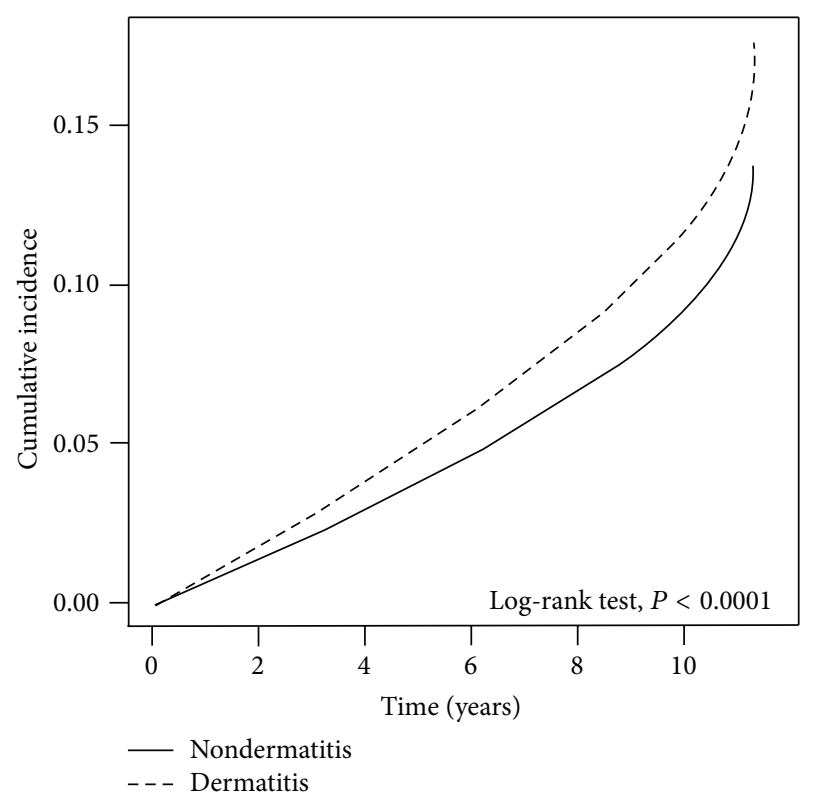

FIGURE 1: The estimated cumulative incidence of stroke between the dermatitis cohort and the nondermatitis cohort by Kaplan-Meier analysis.

Cox's proportional hazard regression demonstrated that the crude hazard ratio (HR) for future stroke among patients with dermatitis was 1.13 (95\% confidence interval, (95\% CI, 1.1-1.16; $P<0.0001)$ and ischemic stroke among patients with dermatitis was 1.16 (95\% CI, 1.12-1.19; $P<0.0001$ ). Furthermore, compared with non-TCM and nonsystemic steroid user, the adjusted hazard ratio (HR) for future stroke among patients treated with only TCM was 1.22 (95\% CI, $1.13-1.32 ; P<0.0001)$, only systemic steroid was 1.55 (95\% CI, 1.43-1.67; $P<0.0001)$ and TCM and systemic steroid user was 1.64 (95\% CI, 1.53-1.76) (Tables 2 and 3). These HR results suggested that dermatitis and patient treated with systemic corticosteroid or TCM may be an independent risk factor for stroke. The log-rank test showed a higher cumulative incidence of ischemic stroke in the patient with dermatitis and treated with systemic corticosteroid group than treated with systemic corticosteroid and TCM, only TCM, and neither systemic corticosteroid nor TCM in the matched cohort during the follow-up period $(P<0.0001$, Figure 2).

We also identified the following independent factors determining the risk of future stroke. The adjusted HRs of stroke were significantly lower in female than male (HR: 0.81; 95\% CI, 0.78-0.83; $P<0.0001)$ and increased with increasing age. Significant adjusted HRs of stroke in Cox proportional hazard models were asthma (HR: 1.07; 95\% CI, 1.02-1.12; $P=0.0073$ ), diabetes mellitus (HR: 1.37; 95\% CI, 1.32-1.41; $P<0.0001$ ), hypertension (HR: 1.87; 95\% CI, 1.81-1.93; $P<$ 0.0001 ), and atrial fibrillation (HR: 1.70; $95 \% \mathrm{CI}, 1.51-1.91$; $P<0.0001)$.

\section{Discussion}

In this large population-based cohort study, we demonstrated that the patient with dermatitis treated with systemic 
TABLE 1: Demographic characteristics and comorbidity in patients with and without dermatitis.

\begin{tabular}{|c|c|c|c|c|c|}
\hline \multirow{3}{*}{ Variables } & \multicolumn{4}{|c|}{ Dermatitis } & \multirow{3}{*}{$P$ value $^{\dagger}$} \\
\hline & \multicolumn{2}{|c|}{$\begin{array}{c}\text { No } \\
(N=235220)\end{array}$} & \multicolumn{2}{|c|}{$\begin{array}{c}\text { Yes } \\
(N=235220)\end{array}$} & \\
\hline & $n$ & $\%$ & $n$ & $\%$ & \\
\hline \multicolumn{6}{|l|}{ Sex } \\
\hline Female & 136734 & 58.13 & 136734 & 58.13 & 0.99 \\
\hline Male & 98486 & 41.87 & 98486 & 41.87 & \\
\hline \multicolumn{6}{|l|}{ Age, years } \\
\hline 20-39 & 118623 & 50.43 & 118623 & 50.43 & 0.99 \\
\hline $40-49$ & 51025 & 21.69 & 51025 & 21.69 & \\
\hline $50-59$ & 31596 & 13.43 & 31596 & 13.43 & \\
\hline$\geq 60$ & 33976 & 14.44 & 33976 & 14.44 & \\
\hline Mean $(\mathrm{SD})^{\dagger}$ & \multicolumn{2}{|c|}{$41.47 \pm 15.92$} & \multicolumn{2}{|c|}{$41.93 \pm 15.52$} & $<0.0001$ \\
\hline \multicolumn{6}{|l|}{ Comorbidity } \\
\hline Asthma & 9394 & 3.99 & 14123 & 6.00 & $<0.0001$ \\
\hline Allergic rhinitis & 21178 & 9.00 & 33243 & 14.13 & $<0.0001$ \\
\hline Urticaria & 11167 & 4.75 & 26983 & 11.47 & $<0.0001$ \\
\hline Diabetes mellitus, DM & 14864 & 6.32 & 20171 & 8.58 & $<0.0001$ \\
\hline Hypertension & 32216 & 13.70 & 40360 & 17.16 & $<0.0001$ \\
\hline Hyperlipidemia & 14819 & 6.30 & 21579 & 9.17 & $<0.0001$ \\
\hline Atrial fibrillation, AF & 625 & 0.27 & 700 & 0.30 & 0.0391 \\
\hline \multicolumn{6}{|l|}{ Treatment } \\
\hline TCM (excluded acupuncture) & 183949 & 78.20 & 207890 & 88.38 & $<0.0001$ \\
\hline Systemic corticosteroid & 160541 & 68.25 & 206402 & 87.75 & $<0.0001$ \\
\hline Dexamethasone & 103693 & 44.08 & 146470 & 62.27 & $<0.0001$ \\
\hline Methyl prednisolone & 35995 & 15.30 & 62122 & 26.41 & $<0.0001$ \\
\hline Prednisolone & 128167 & 54.49 & 182906 & 77.76 & $<0.0001$ \\
\hline \multicolumn{6}{|l|}{ Treatment (new) } \\
\hline Non-TCM and nonsystemic steroid & 25312 & 10.76 & 5507 & 2.34 & $<0.0001$ \\
\hline Only TCM & 49367 & 20.99 & 23311 & 9.91 & \\
\hline Only systemic steroid & 25959 & 11.04 & 21823 & 9.28 & \\
\hline TCM and systemic steroid & 134582 & 57.22 & 184579 & 78.47 & \\
\hline \multicolumn{6}{|l|}{ Outcome } \\
\hline Stroke & 10006 & 4.25 & 13079 & 5.56 & $<0.0001$ \\
\hline Hemorrhagic stroke & 1543 & 0.66 & 1463 & 0.62 & 0.1432 \\
\hline Ischemic stroke & 9277 & 3.94 & 12450 & 5.29 & $<0.0001$ \\
\hline
\end{tabular}

Chi-square test; ${ }^{\dagger}$ two-sample $t$-test.

corticosteroid is a risk factor for stroke and the patients treated with TCM may decrease incidence of this risk. Patients with dermatitis treated with TCM had decreased incidence of ischemic stroke compared with the corticosteroid group. These findings support the concept that dermatitis may exert a systemic effect contributing to stroke and different treatments are important confounders.

Corticosteroid is the mainstay treatment for dermatitis, with the route of administration and dosage schedule dependent primarily on the severity, while complications range in severity and frequency, which are generally considered to be depend on the dosage and/or duration of corticosteroid. However, the adverse effects result from not only the cumulative corticosteroid dose but also high-dose corticosteroid treatment which was significantly associated with development of stroke [18]. Obesity, diabetes mellitus, hypertension, atrial fibrillation, and hyperlipidemia may be worse as corticosteroid treated; furthermore, these adverse effects may contribute to the later development of atherosclerosis and ischemic stroke [19-21].

The atherosclerotic changes or stroke are associated with inflammatory processes resulting from several dermatoses, such as atopic dermatitis [8], dermatitis herpetiformis [22], systemic lupus erythematosus [23], bullous pemphigoid [24], drug rash eosinophilia and systemic symptoms (DRESS) [25], and psoriasis [26]. Possible explanations for the high risk of stroke in patients with dermatitis are atherosclerotic changes [4, 14, 23], oxidative stress [27], and activation of the coagulation system related to chronic inflammation $[28,29]$. Increasing evidence has shown that systemic inflammation 
TABLE 2: Cox model measured hazard ratio and 95\% confidence intervals of stroke associated with dermatitis and covariates.

\begin{tabular}{|c|c|c|c|c|c|c|}
\hline \multirow{2}{*}{ Characteristics } & \multicolumn{3}{|c|}{ Crude } & \multicolumn{3}{|c|}{ Adjusted } \\
\hline & HR & $(95 \% \mathrm{CI})$ & $P$ value & HR & $(95 \% \mathrm{CI})$ & $P$ value \\
\hline \multicolumn{7}{|l|}{ Dermatitis $(\mathrm{ref}=\mathrm{no})$} \\
\hline Yes & 1.27 & $(1.23-1.3)$ & $<0.0001$ & 1.13 & $(1.10-1.16)$ & $<0.0001$ \\
\hline \multicolumn{7}{|l|}{ Gender $($ ref = male $)$} \\
\hline Female & 0.67 & $(0.66-0.69)$ & $<0.0001$ & 0.79 & $(0.77-0.81)$ & $<0.0001$ \\
\hline \multicolumn{7}{|l|}{ Age, years $($ ref = 20-39) } \\
\hline $40-49$ & 4.18 & $(3.97-4.41)$ & $<0.0001$ & 3.75 & $(3.56-3.96)$ & $<0.0001$ \\
\hline $50-59$ & 9.58 & $(9.1-10.08)$ & $<0.0001$ & 7.27 & $(6.90-7.67)$ & $<0.0001$ \\
\hline$\geq 60$ & 25.70 & $(24.54-26.92)$ & $<0.0001$ & 16.17 & $(15.37-17.00)$ & $<0.0001$ \\
\hline \multicolumn{7}{|l|}{ Comorbidity } \\
\hline \multicolumn{7}{|l|}{ Asthma $(\mathrm{ref}=\mathrm{no})$} \\
\hline Yes & 2.42 & $(2.32-2.53)$ & $<0.0001$ & 1.07 & $(1.03-1.12)$ & 0.0019 \\
\hline \multicolumn{7}{|l|}{ Allergic rhinitis $(\mathrm{ref}=\mathrm{no})$} \\
\hline Yes & 1.02 & $(0.97-1.06)$ & 0.4694 & 0.95 & $(0.91-0.99)$ & 0.0158 \\
\hline \multicolumn{7}{|l|}{ Urticaria $($ ref $=$ no $)$} \\
\hline Yes & 1.12 & $(1.07-1.17)$ & $<0.0001$ & 0.99 & $(0.94-1.04)$ & 0.6715 \\
\hline \multicolumn{7}{|l|}{$\mathrm{DM}(\mathrm{ref}=\mathrm{no})$} \\
\hline Yes & 4.20 & $(4.07-4.33)$ & $<0.0001$ & 1.35 & $(1.30-1.39)$ & $<0.0001$ \\
\hline \multicolumn{7}{|l|}{ Hypertension $(\mathrm{ref}=\mathrm{no})$} \\
\hline Yes & 6.26 & $(6.1-6.43)$ & $<0.0001$ & 1.87 & $(1.82-1.93)$ & $<0.0001$ \\
\hline \multicolumn{7}{|l|}{ Hyperlipidemia $($ ref = no $)$} \\
\hline Yes & 3.08 & $(2.97-3.18)$ & $<0.0001$ & 1.00 & $(0.96-1.04)$ & 0.9719 \\
\hline \multicolumn{7}{|l|}{$\mathrm{AF}(\mathrm{ref}=\mathrm{no})$} \\
\hline Yes & 7.04 & $(6.28-7.9)$ & $<0.0001$ & 1.68 & $(1.50-1.89)$ & $<0.0001$ \\
\hline \multicolumn{7}{|c|}{ Treatment (ref = non-TCM and nonsystemic steroid $)$} \\
\hline Only TCM & 0.98 & $(0.90-1.06)$ & 0.5871 & 1.22 & $(1.13-1.32)$ & $<0.0001$ \\
\hline Only systemic steroid & 2.19 & $(2.03-2.36)$ & $<0.0001$ & 1.55 & $(1.43-1.67)$ & $<0.0001$ \\
\hline TCM and systemic steroid & 1.66 & $(1.55-1.77)$ & $<0.0001$ & 1.64 & $(1.53-1.76)$ & $<0.0001$ \\
\hline
\end{tabular}

HR: hazard ratio; $\mathrm{CI}$ : confidence interval.

Adjusted HR: adjusted for age, gender, treatment, and comorbidity in Cox proportional hazards regression.

can promote the progression of atherosclerosis and thrombosis to ischemic stroke [6]. There are several possible mechanisms of dermatitis resulting in stroke. First, the elevation of platelet activation and reducing fibrinolysis were founded in patients with chronic inflammatory allergic diseases such as atopic dermatitis $[30,31]$. Second, mast cell may participate in atherosclerosis by releasing proinflammatory cytokines, chemokines, and proteases to induced inflammatory cell recruitment, cell apoptosis, and angiogenesis [32, 33]. Third, increased serum IgE levels in myocardial infarction patients and mast cell accumulated in atherosclerotic lesions [34]. Fourth, hypereosinophilia may play an import role in some of these dermatoses, included dermatitis, bullous pemphigoid, and DRESS. Thrombosis may be related to eosinophilic hypothiocyanous acid productions, which lead to a prothrombotic state [35]. Furthermore, encephalopathy may arise from small cerebral stroke or direct eosinophil toxicity [36]. Dermatitis is an allergic disease, like asthma; it probably exerts systemic inflammatory effect in a similar fashion, thereby contributing to cardiovascular or cerebrovascular consequences. However, allergic rhinitis and urticarial seem to be of milder and less systemic inflammation than other atopic diseases.
A number of studies on TCM have been performed, with a collective result of symptom improvement and decreased levels of inflammatory cytokines. Since standard TCM prescribed of many herbs combined in different forms and dosed differently depending on each individual patient, randomized control trials in this area have been difficult to perform. It has been postulated that Zemaphyte might work as an efficient antioxidant, capable of scavenging both superoxide and hydroxyl and preventing peroxidation of biological membranes. Pentaherbs formulation, another TCM prescription formula, postulated that suppression of the low-affinity receptors for IgE on antigen-presenting cell, modulated mast cells and inhibited the inflammatory mediators from mast cells [37], and possessed immunomodulatory effects and inflammatory mediators [38]. Methodological advantages of the interdisciplinary secondary database utilized include a high degree of generalizability, completeness and absence of recall bias due to prospective input of diagnoses and research questions and large sample size [39]. In this study, we replicated the previous reported positive association between the major stroke risk factors and found that atrial fibrillation, hypertension, diabetes, and hyperlipidemia seem to be very sensitive to change to multivariate models (Table 2). This may 
TABLE 3: Cox model measured hazard ratio and 95\% confidence intervals of ischemic stroke associated with dermatitis and covariates.

\begin{tabular}{|c|c|c|c|c|c|c|}
\hline \multirow{2}{*}{ Characteristics } & \multicolumn{3}{|c|}{ Crude } & \multicolumn{3}{|c|}{ Adjusted } \\
\hline & HR & $(95 \% \mathrm{CI})$ & $P$ value & HR & $(95 \% \mathrm{CI})$ & $P$ value \\
\hline \multicolumn{7}{|l|}{ Dermatitis $(\mathrm{ref}=\mathrm{no})$} \\
\hline Yes & 1.30 & $(1.27-1.34)$ & $<0.0001$ & 1.16 & $(1.12-1.19)$ & $<0.0001$ \\
\hline \multicolumn{7}{|l|}{ Gender $($ ref $=$ male $)$} \\
\hline Female & 0.69 & $(0.67-0.71)$ & $<0.0001$ & 0.81 & $(0.78-0.83)$ & $<0.0001$ \\
\hline \multicolumn{7}{|l|}{ Age, years $($ ref $=20-39)$} \\
\hline $40-49$ & 4.60 & $(4.34-4.87)$ & $<0.0001$ & 4.12 & $(3.89-4.37)$ & $<0.0001$ \\
\hline $50-59$ & 10.88 & $(10.3-11.5)$ & $<0.0001$ & 8.25 & $(7.8-8.73)$ & $<0.0001$ \\
\hline$\geq 60$ & 29.39 & $(27.95-30.89)$ & $<0.0001$ & 18.48 & $(17.5-19.5)$ & $<0.0001$ \\
\hline \multicolumn{7}{|l|}{ Comorbidity } \\
\hline \multicolumn{7}{|l|}{ Asthma $(\mathrm{ref}=\mathrm{no})$} \\
\hline Yes & 2.45 & $(2.35-2.57)$ & $<0.0001$ & 1.07 & $(1.02-1.12)$ & 0.0073 \\
\hline \multicolumn{7}{|l|}{ Allergic rhinitis $(\mathrm{ref}=\mathrm{no})$} \\
\hline Yes & 1.04 & $(1-1.09)$ & 0.0829 & 0.97 & $(0.92-1.01)$ & 0.1195 \\
\hline \multicolumn{7}{|l|}{ Urticaria $($ ref $=$ no $)$} \\
\hline Yes & 1.12 & $(1.07-1.18)$ & $<0.0001$ & 0.98 & $(0.94-1.03)$ & 0.5232 \\
\hline \multicolumn{7}{|l|}{$\mathrm{DM}(\mathrm{ref}=\mathrm{no})$} \\
\hline Yes & 4.35 & $(4.22-4.49)$ & $<0.0001$ & 1.37 & $(1.32-1.41)$ & $<0.0001$ \\
\hline \multicolumn{7}{|l|}{ Hypertension $(\mathrm{ref}=\mathrm{no})$} \\
\hline Yes & 6.48 & $(6.31-6.65)$ & $<0.0001$ & 1.87 & $(1.81-1.93)$ & $<0.0001$ \\
\hline \multicolumn{7}{|l|}{ Hyperlipidemia $($ ref = no) } \\
\hline Yes & 3.18 & $(3.07-3.3)$ & $<0.0001$ & 1.01 & $(0.97-1.05)$ & 0.6112 \\
\hline \multicolumn{7}{|l|}{$\mathrm{AF}(\mathrm{ref}=\mathrm{no})$} \\
\hline Yes & 7.24 & $(6.44-8.14)$ & $<0.0001$ & 1.70 & $(1.51-1.91)$ & $<0.0001$ \\
\hline \multicolumn{7}{|c|}{ Treatment $(\mathrm{ref}=$ non-TCM and nonsystemic steroid) } \\
\hline Only TCM & 1.01 & $(0.93-1.09)$ & 0.8967 & 1.25 & $(1.15-1.36)$ & $<0.0001$ \\
\hline Only systemic steroid & 2.23 & $(2.06-2.42)$ & $<0.0001$ & 1.56 & $(1.44-1.69)$ & $<0.0001$ \\
\hline TCM and systemic steroid & 1.72 & $(1.60-1.85)$ & $<0.0001$ & 1.68 & $(1.57-1.81)$ & $<0.0001$ \\
\hline
\end{tabular}

HR: hazard ratio; $\mathrm{CI}$ : confidence interval.

Adjusted HR: adjusted for age, gender, treatment, and comorbidity in Cox proportional hazards regression.

be due to the low prevalence of those traditional risk factors for stroke in the dermatitis group. We demonstrated that dermatitis may be an independent risk factor for ischemic stroke. In light of our limited understanding of the exact mechanisms explaining the adverse stroke risk factors in dermatitis patients, it has been speculated that the established association between stroke and different treatments might explain these findings.

Our study has several limitations. First, patients with dermatitis and stroke were identified using a diagnostic code in a database, introducing the possibility of misclassification because of coding errors or misdiagnosis. Second, some potential risk factors, including obesity, smoking, alcohol use, and family history of cardiovascular disease, were not included in our analyses because these data were not available. Third, the follow-up period may not have been sufficiently long to detect stroke development because atopic dermatitis always course in child to teenager but stroke often attacks Middle-aged to elderly patients. Fourth, we could not directly evaluate the severity of dermatitis stroke, the accumulated dosage of systemic corticosteroid, ingredients of TCM, and each comorbidity. Finally, because we did not have the information of causes of death, stroke may be a cause of death but was not recorded as an endpoint. The role of inflammation biomarkers, ingredients of TCM, and the relationship between TCM, dermatitis, and stroke are not clear. Further research is needed to determine the possible pathogenic mechanisms of TCM prescribed in dermatitis and stroke which is necessary.

\section{Conclusions}

This large population-based study demonstrated that patients treated with systemic corticosteroid had an increased risk of stroke and that the risk probably decreased by TCM treatment.

\section{Conflict of Interests}

The authors declare that they have no conflict of interests.

\section{Acknowledgments}

This study is supported in part by China Medical University (CMU103-S-47), CMU under the Aim for Top University Plan of the Taiwan Ministry of Education, Taiwan Ministry 


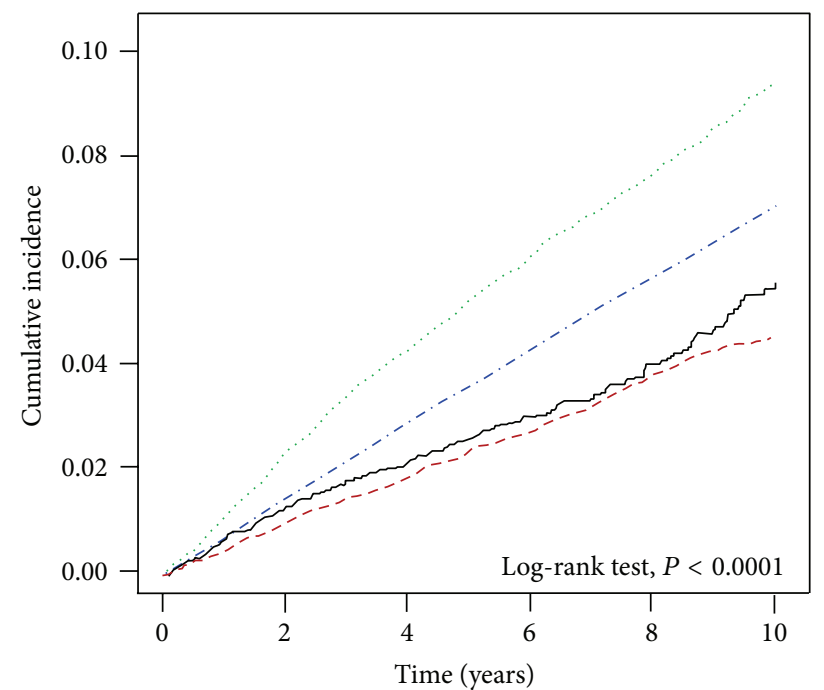

- Non-TCM and nonsystemic steroid ... - TCM and systemic steroid

Only systemic steroid Only TCM

FIGURE 2: The estimated cumulative incidence of ischemic stroke between those treated with CTM or systemic corticosteroid in the patients of dermatitis cohort by Kaplan-Meier analysis.

of Health and Welfare Clinical Trial and Research Center of Excellence (104-TDU-B-212-113002), Academia Sinica Taiwan Biobank, Stroke Biosignature Project (BM104010096), NRPB Stroke Clinical Trial Consortium (MOST 103-2325B-039-006), Tseng-Lien Lin Foundation, Taichung, Taiwan Brain Disease Foundation, and Katsuzo and Kiyo Aoshima Memorial Funds, Japan.

\section{References}

[1] C. K. Kim, D. H. Kim, M. S. Lee, J. I. Kim, L. S. Wieland, and B. C. Shin, "Randomized controlled trials on complementary and traditional medicine in the korean literature," Evidence-Based Complementary and Alternative Medicine, vol. 2014, Article ID 194047, 10 pages, 2014.

[2] C. Wu, C. Wang, M. Tsai, W. Huang, and J. Kennedy, "Trend and pattern of herb and supplement use in the United States: results from the 2002, 2007, and 2012 national health interview surveys," Evidence-Based Complementary and Alternative Medicine, vol. 2014, Article ID 872320, 7 pages, 2014.

[3] M. Steinhoff, A. Steinhoff, B. Homey, T. A. Luger, and S. W. Schneider, "Role of vasculature in atopic dermatitis," The Journal of Allergy and Clinical Immunology, vol. 118, no. 1, pp. 190-197, 2006.

[4] Y. Wang, H. Gao, C. M. Loyd et al., "Chronic skin-specific inflammation promotes vascular inflammation and thrombosis," Journal of Investigative Dermatology, vol. 132, no. 8, pp. 2067-2075, 2012.

[5] N. Shenoy, K. A. Shenoy, and U. P. Ratnakar, "The perceptual preferences in learning among dental students in clinical subjects," Journal of Clinical and Diagnostic Research, vol. 7, no. 8, pp. 1683-1685, 2013.

[6] M. S. V. Elkind, "Inflammatory markers and stroke," Current Cardiology Reports, vol. 11, no. 1, pp. 12-20, 2009.
[7] L. L. N. Husemoen, T. Skaaby, T. Jørgensen et al., "No association between loss-of-function mutations in filaggrin and diabetes, cardiovascular disease, and all-cause mortality," PLoS ONE, vol. 8, no. 12, Article ID e84293, 2013.

[8] V. Y.-F. Su, T.-J. Chen, C.-M. Yeh et al., "Atopic dermatitis and risk of ischemic stroke: a nationwide population-based study," Annals of Medicine, vol. 46, no. 2, pp. 84-89, 2014.

[9] F. Formiga, J. F. Meco, X. Pinto, J. Jacob, I. Moga, and R. Pujol, "Lipid and lipoprotein levels in premenopausal systemic lupus erythematosus patients," Lupus, vol. 10, no. 5, pp. 359-363, 2001.

[10] B. F. A. Freire, R. C. da Silva, A. T. Fabro, and D. C. dos Santos, "Is systemic lupus erithematosus a new risk factor for atherosclerosis?" Arquivos Brasileiros de Cardiologia, vol. 87, no. 3, pp. 300-306, 2006.

[11] K.-L. E. Hon, T. F. Leung, H. C. Yau, and T. Chan, "Paradoxical use of oral and topical steroids in steroid-phobic patients resorting to traditional Chinese medicines," World Journal of Pediatrics, vol. 8, no. 3, pp. 263-267, 2012.

[12] J. I. Silverberg, M. Lee-Wong, and N. B. Silverberg, "Complementary and alternative medicines and childhood eczema: a US population-based study," Dermatitis, vol. 25, no. 5, pp. 246-254, 2014.

[13] K.-S. Tsai, T.-C. Lin, M.-T. Wu et al., "Irritant contact dermatitis risk of common topical traditional chinese medicines used for skin-lightening: a pilot clinical trial with 30 volunteers," Evidence-Based Complementary and Alternative Medicine, vol. 2014, Article ID 609064, 8 pages, 2014.

[14] P. E. Harris, K. L. Cooper, C. Relton, and K. J. Thomas, "Prevalence of complementary and alternative medicine (CAM) use by the general population: a systematic review and update," International Journal of Clinical Practice, vol. 66, no. 10, pp. 924939, 2012.

[15] H. Y. Chen, Y. C. Chang, C. C. Lin, F. C. Sung, and W. C. Chen, "Obstructive sleep apnea patients having surgery are less associated with glaucoma," Journal of Ophthalmology, vol. 2014, Article ID 838912, 6 pages, 2014.

[16] H.-Y. Chen, Y.-C. Chang, I.-J. Wang, and W.-C. Chen, "Comparison of glaucoma diagnoses using Stratus and Cirrus optical coherence tomography in different glaucoma types in a Chinese population," Journal of Glaucoma, vol. 22, no. 8, pp. 638-646, 2013.

[17] S. Wacholder, J. K. McLaughlin, D. T. Silverman, and J. S. Mandel, "Selection of controls in case-control studies. I. Principles," American Journal of Epidemiology, vol. 135, no. 9, pp. 1019-1028, 1992.

[18] T. F. Leung, K. Y. Wong, C. K. Wong et al., "In vitro and clinical immunomodulatory effects of a novel Pentaherbs concoction for atopic dermatitis," British Journal of Dermatology, vol. 158, no. 6, pp. 1216-1223, 2008.

[19] A. Zonana-Nacach, S. G. Barr, L. S. Magder, and M. Petri, "Damage in systemic lupus erythematosus and its association with corticosteroids," Arthritis and Rheumatism, vol. 43, no. 8, pp. 1801-1808, 2000.

[20] L. Wei, T. M. MacDonald, and B. R. Walker, "Taking glucocorticoids by prescription is associated with subsequent cardiovascular disease," Annals of Internal Medicine, vol. 141, no. 10, pp. 764-770, 2004.

[21] C. F. Christiansen, S. Christensen, F. Mehnert, S. R. Cummings, R. D. Chapurlat, and H. T. Sørensen, "Glucocorticoid use and risk of atrial fibrillation or flutter: A population-based, casecontrol study," Archives of Internal Medicine, vol. 169, no. 18, pp. 1677-1683, 2009. 
[22] J. T. Lear, R. H. Neary, P. Jones, D. A. Fitzgerald, and J. S. C. English, "Risk factors for ischaemic heart disease in patients with dermatitis herpetiformis," Journal of the Royal Society of Medicine, vol. 90, no. 5, pp. 247-249, 1997.

[23] I. N. Bruce, “Not only...but also': factors that contribute to accelerated atherosclerosis and premature coronary heart disease in systemic lupus erythematosus," Rheumatology, vol. 44, no. 12, pp. 1492-1502, 2005.

[24] Y. J. Chen, C. Y. Wu, M. W. Lin et al., "Comorbidity profiles among patients with bullous pemphigoid: a nationwide population-based study," British Journal of Dermatology, vol. 165, no. 3, pp. 593-599, 2011.

[25] R. Cahyanur, D. Oktavia, and S. Koesno, "DRESS and Ischemic Stroke," Acta Medica Indonesiana, vol. 44, no. 3, pp. 239-242, 2012.

[26] C.-H. Chiang, C.-C. Huang, W.-L. Chan et al., "Psoriasis and increased risk of ischemic stroke in Taiwan: a nationwide study," Journal of Dermatology, vol. 39, no. 3, pp. 279-281, 2012.

[27] N. Sivaranjani, S. Venkata Rao, and G. Rajeev, "Role of reactive oxygen species and antioxidants in atopic dermatitis," Journal of Clinical and Diagnostic Research, vol. 7, no. 12, pp. 2683-2685, 2013.

[28] S. C. Pitchford, H. Yano, R. Lever et al., "Platelets are essential for leukocyte recruitment in allergic inflammation," Journal of Allergy and Clinical Immunology, vol. 112, no. 1, pp. 109-118, 2003.

[29] M. Gawaz, H. Langer, and A. E. May, "Platelets in inflammation and atherogenesis," Journal of Clinical Investigation, vol. 115, no. 12, pp. 3378-3384, 2005.

[30] R. Tamagawa-Mineoka, N. Katoh, E. Ueda, K. Masuda, and S. Kishimoto, "Platelet-derived microparticles and soluble Pselectin as platelet activation markers in patients with atopic dermatitis," Clinical Immunology, vol. 131, no. 3, pp. 495-500, 2009.

[31] M. Nastalek, A. Wojas-Pelc, and A. Undas, "Plasma fibrin clot properties in atopic dermatitis: links between thrombosis and atopy," Journal of Thrombosis and Thrombolysis, vol. 30, no. 2, pp. 121-126, 2010.

[32] J. Sun, G. K. Sukhova, P. J. Wolters et al., "Mast cells promote atherosclerosis by releasing proinflammatory cytokines," Nature Medicine, vol. 13, no. 6, pp. 719-724, 2007.

[33] M. Ihara, H. Urata, A. Kinoshita et al., "Increased chymasedependent angiotensin II formation in human atherosclerotic aorta," Hypertension, vol. 33, no. 6, pp. 1399-1405, 1999.

[34] M. Jeziorska, C. Mccollum, and D. E. Woolley, "Mast cell distribution, activation, and phenotype in atherosclerotic lesions of human carotid arteries," Journal of Pathology, vol. 182, no. 1, pp. 115-122, 1997.

[35] J.-G. Wang, S. A. Mahmud, J. A. Thompson, J.-G. Geng, N. S. Key, and A. Slungaard, "The principal eosinophil peroxidase product, $\mathrm{HOSCN}$, is a uniquely potent phagocyte oxidant inducer of endothelial cell tissue factor activity: a potential mechanism for thrombosis in eosinophilic inflammatory states," Blood, vol. 107, no. 2, pp. 558-565, 2006.

[36] P. W. Kaplan, "Central nervous system problems with eosinophilia," Archives of Neurology, vol. 68, no. 12, pp. 1613-1614, 2011.

[37] L. Y. Chung, "Antioxidant profiles of a prepared extract of Chinese herbs for the treatment of atopic eczema," Phytotherapy Research, vol. 22, no. 4, pp. 493-499, 2008.

[38] B. C. L. Chan, K. L. E. Hon, P. C. Leung et al., "Traditional Chinese medicine for atopic eczema: pentaHerbs formula suppresses inflammatory mediators release from mast cells," Journal of Ethnopharmacology, vol. 120, no. 1, pp. 85-91, 2008.

[39] H. T. Sørensen, S. Sabroe, and J. Olsen, "A framework for evaluation of secondary data sources for epidemiological research," International Journal of Epidemiology, vol. 25, no. 2, pp. 435442, 1996. 


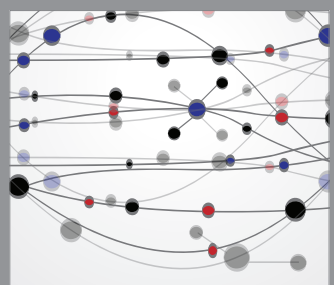

The Scientific World Journal
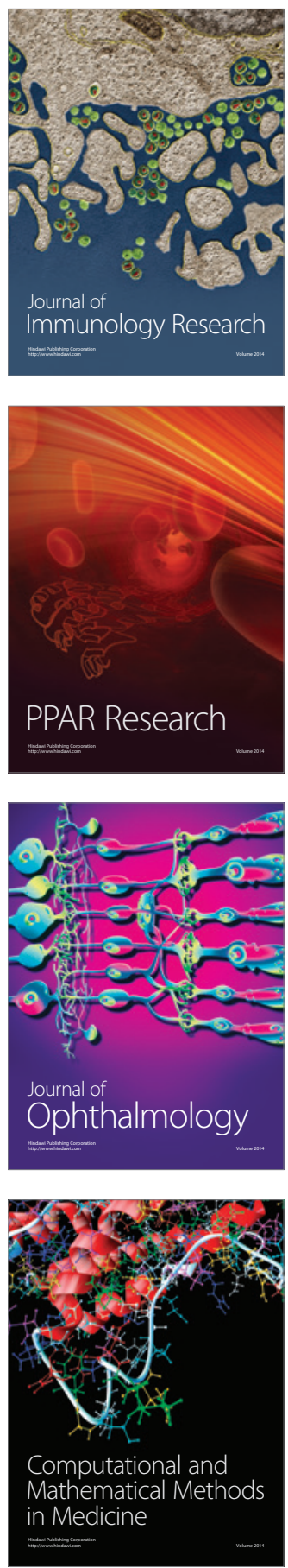

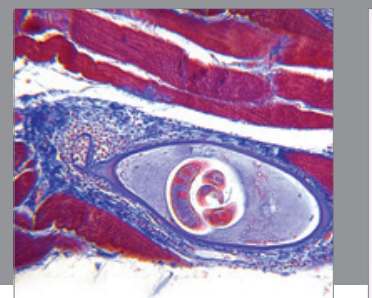

Gastroenterology

Research and Practice
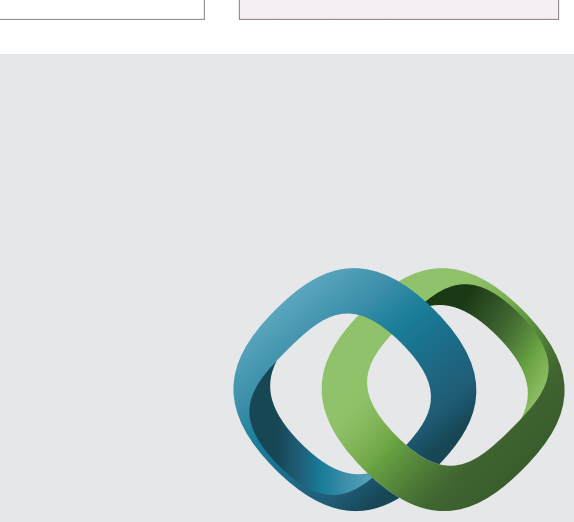

\section{Hindawi}

Submit your manuscripts at

http://www.hindawi.com
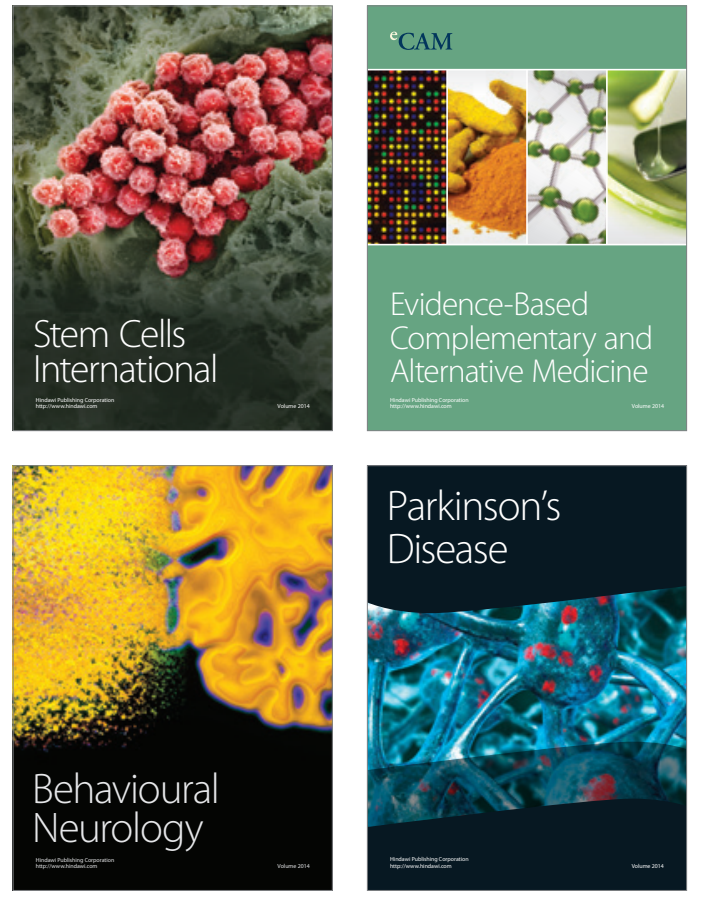
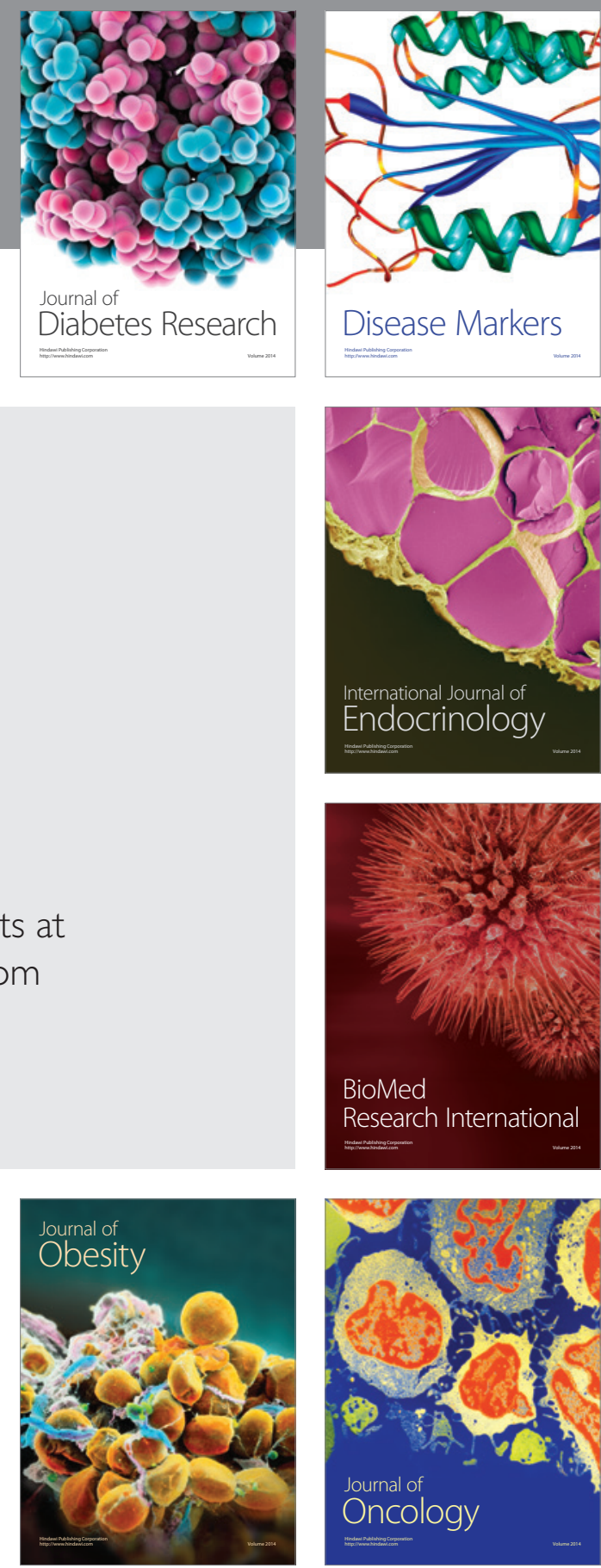

Disease Markers
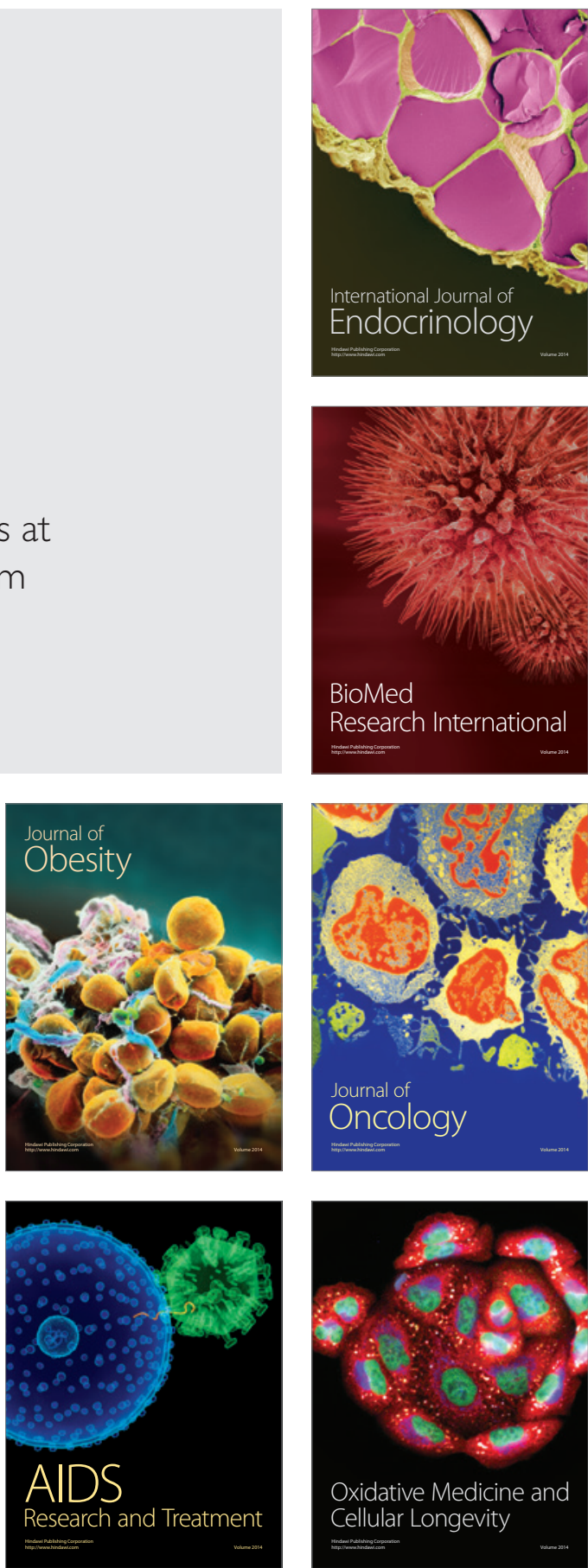